\title{
QUALITY CONTROL OF A MILLING PROCESS USING PROCESS DATA MANAGEMENT IN THE AEROSPACE INDUSTRY
}

\author{
D. Pfirrmann ${ }^{1 *}$, M. Voit ${ }^{1}$, M. Eckstein ${ }^{1}$ \\ ${ }^{1}$ MTU Aero Engines AG, Munich, Germany \\ *Corresponding author; e-mail: daniel.pfirrmann-external@mtu.de
}

\begin{abstract}
Global production is in a state of change, with multiple challenges. One challenge is the digitization of production processes in the aerospace industry, due to the high requirements regarding the reliability of the transportation vehicle. It is a premise to record the manufacturing processes using sensors and to analyze them by means of process data management. Therefore, approaches regarding the predictable quality of the manufacturing process and the predictable maintenance of the machinery offer a high industrial potential. The aim should be to eliminate any testing processes to obtain an early statement about the component quality at an early stage in the value chain by means of process data management. Furthermore, based on the collected process data, the production process can be controlled preventively during the active analysis. For this, appropriate algorithms are necessary that allow reliable process statement and control.

This publication presents the views of an aerospace industry company in terms of requirements and possible approaches to process data management. As application examples, the challenges and a solution concept for a milling process will be explained, with the claim that the process data management is applicable to different manufacturing processes of the value chain.
\end{abstract}

\section{Keywords:}

Process data management; Milling; Predictive quality; Predictive maintenance; Aerospace industry

\section{INTRODUCTION}

In the course of fully automated production, resourceefficient production is becoming increasingly important [Reinhardt et al. 2012]. The systems must have high availability and production errors, such as tool failure, should be avoided. The digitization of the manufacturing industry offers some possibilities to avoid production errors [Hofmann 2017]. Especially in the aerospace industry, unrecognized production errors can lead to far-reaching consequences. For this reason, it is the premise to further support the traceability of the component quality on the basis of the digitization of the manufacturing industry. As an example of this, the many measuring and testing operations during and after milling can be cited, which are necessary due to the requirements of the aerospace industry.

Process deviations lead in the aerospace industry to component rejects which are generally very expensive, since the machine and personnel costs are very high [Lin 2013]. Process deviations are caused among other things by tool wear, defects of the raw material and external influences [Tönshoff 2002]. This example shows that there is a high demand for predictable part quality, predictable machine maintenance and preventative process control due to the digitalization of the manufacturing. Edge computing offers many advantages and is considered as a requirement for the previously mentioned production possibilities. According to [Shi et al. 2016] Edge Computing is the decentralized data processing at the interfaces of the network. The collected data is partially processed at the end device. In manufacturing machines, the sensor and data acquisition system is a key aspect for Edge Computing. This approach is explained in more detail in chapter 3 . The possibilities of process control by Edge Computing are explained in more detail below. Edge Computing needs further research as industry, especially the aerospace industry, sees great benefits and needs in it. Therefore predictive part quality, predictive machine maintenance and predictive process control are part of the Edge Computing. Predictive part quality is the statement about the quality of a manufacturing process based on the process parameters and the resulting component quality during the manufacturing process. Predictive machine maintenance is the prediction of possible maintenance intervals and machine breakdowns of the production machine as well as upcoming tool changes. This machine data must be determined during the manufacturing process using live data. A process control loop, which results from the recorded process data, is understood as a preventive process control. Here, the production process is regulated on the basis of the recorded data in real time so that any incorrect influences can be eliminated. The initial industrial approach is to obtain a statement about the state of the manufacturing process. For this a statement about the stability of the process must be made. The premise is to record the process data with sufficient accuracy using suitable sensors. These data should be collected at the highest possible frequency for a detailed process statement. Furthermore, a suitable interface for the 
acquisition of the machine data is necessary. For the implementation of preventive process control, it is still necessary for the software to analyze the machine data in real time and, if required, to incorporate corrected machine values for countermeasures. The data must be recorded along the production process chain. This approach is the most extensive challenge, but it is necessary for competitive production in Germany as a high-wage location. Figure 1 illustrates the correlation outlined.

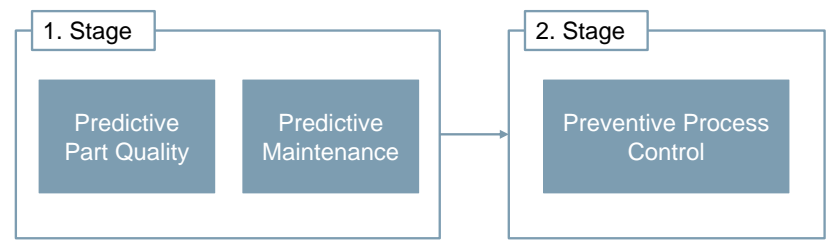

Fig. 1: Correlation between predictive part quality, predictive maintenance and preventive process control.

The following content deals with an industrial approach for the process data management of a milling process. It will highlight challenges and the industrial approach to process data management and provide an outlook for the aerospace industry.

\section{CHALLENGES OF COMMERCIAL AIRCRAFT ENGINES}

The preferred design of present-day jet-engines for commercial aircraft is the turbofan [Gunston ${ }^{\circ} 1996$ ], [Bräunling ${ }^{\circ} 2009$ ]. Here, the mayor part of the propulsive thrust is not generated by the exhaust gas, exiting the turbine but by the rotation of a shrouded fan, which together with the compressor - is powered by the turbine. With the very recent generation of turbofans airlines can increase the energy efficiency of their fleet by an additional $15-20 \%$. The geared turbofan allows the fan and all sections of the complete compressor-turbine assembly to rotate at its optimum aerodynamic speed thus reducing both fuel burn and noise.

To utilize the full potential of this engine design it is important to choose latest materials like high strength Titanium and Ni-superalloys. These materials are sensitive to material imperfections and require an aligned sequence of master forming, forging and subsequent manufacturing to final shape. Statistical data provided by the Federal Aviation Administration (FAA) shows very clearly, that besides the human factor the main technical source for inflight shutdown of aeroengines today are imperfections caused by manufacturing. A very drastic example for the impact of manufacturing on engine life is the so called "Pensacola Incident" [NTS ${ }^{\circ} 1996$ ]. The reason for this uncontained failure was the misjudgment of a tool break during pre-drilling of compressor disk. Tiny pieces of the broken tool remained in a bolthole causing the death of two passengers by debris of a broken compressor disk after 10 years in service.

Today, the robustness of the manufacturing is considered for the calculation of the safe life of rotating engine hardware. A system of credits for the application of latest material tests and effective monitoring technology during manufacturing ensure, that imperfections, that can occur along the whole manufacturing chain at any time are all detected and their possible impact on the corresponding component is investigated. This know-how has already become more and more important during the last two decades and will be most essential for the future.
Although air traffic is already operating on a very high level of safety, enginemakers are well aware, that in times of increasing air traffic a corresponding number of engineshutdowns are not acceptable for passengers and airlines. This challenge can only be mastered with an improved control over the excellence of the manufacturing process. An important instrument could be the monitoring of chipmaking processes. [Eckstein ${ }^{\circ} 2013$ ]

Until today, major rotor parts of jet-engines are predominantly machined by conventional chipmaking methods like turning, milling, drilling and grinding.

\section{PROCESS DATA MANAGEMENT USING THE EXAMPLE OF A MILLING PROCESS IN THE AVIATION INDUSTRY}

The machining of nickel and titanium materials poses considerable challenges for machining production. Both materials are considered to be difficult to machine and highly abrasive, which has a significant impact on tool life. In particular, the high flexibility of the material, which also has an increased susceptibility to vibrations, is particularly evident in titanium.

This fact makes machining, especially in the field of aviation, a challenge due to the high requirements for position and form tolerances of the components that apply here. Since components which are used, for example, in an engine are designed due to the high thermal and mechanical alternating loads in a precise range of fatigue strength, this also results in high demands on the quality of the workpieces. In particular, special attention is paid to the surface integrity of the components to ensure a long product life of the engine components.

In the area of engine components, MTU Aero Engines AG specializes in the high-pressure compressor, the lowpressure turbine and parts of the housing.

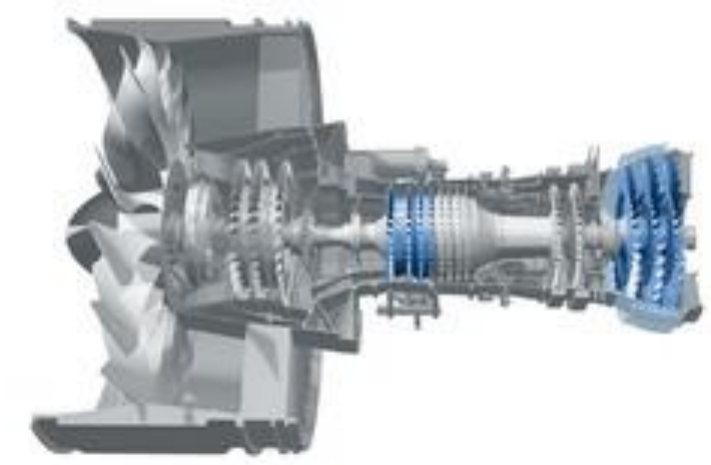

Fig. 2: Profile of an engine and marking of parts manufactured by MTU (blue) [MTU 2019].

Especially the parts of the high-pressure compressor make themselves extremely difficult to produce by the blisk design used here. The difficult freeform surfaces of the blade geometries, seen in figure 3 , are milled directly from the solid, which sometimes leads to very long processing times. 


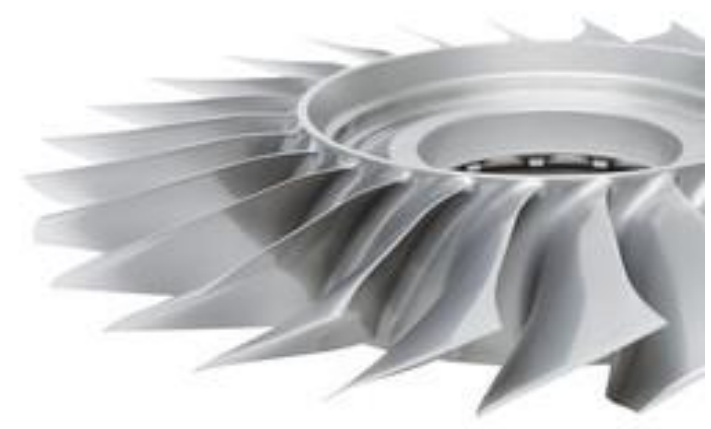

Fig. 3: High pressure compressor Blisk [MTU 2019].

This poses particular problems for process monitoring and the associated process data management. The long processing time generates the correspondingly high amounts of data that need to be processed and saved.

The inclusion of process data is made even more difficult by the conditions in mass production. This is where the problems of data generation begin. External sensor technology is difficult to accommodate in a process-related location without disturbing the processing. In addition, data processing must under no circumstances burden the NCU (Numerical Controller Unit), as the quality of the components must not be degraded. In addition, if one has integrated a system for data acquisition, the quality of the data. During the finishing process of a blisk, only extremely little material removal is achieved, which makes the analysis more difficult due to the strong noise caused by the machine itself. However, the finishing process is crucial for the quality statement about the component, since this produces the final contour.

Once this hurdle has been overcome, the requirements for the sample rate must be defined. Is it necessary or possible to process the data in real time? It must also be clarified which frequency range is to be regarded as real time. The pressure or the flow rate of coolants can be scanned in the range of a few $\mathrm{Hz}$ without losing relevant data because these measurements act very sluggish, but if the vibration of a milling spindle should be monitored it can quickly get in the 2-digit $\mathrm{kHz}$ range to collect all relevant data. Thus, the meaningfulness of the data depends heavily on the process and the definition of real time used here.

If a process is controlled using sensor data, fast data processing and a short reaction time are essential. Here, however, there is another issue to consider in the aviation industry. Critical components, that can lead to a failure of the engine in case of failure, are tested in production after their development. When the quality of the workpiece matches the high quality standards in relation of surface and shape accuracy, the entire process is frozen. This means that the tool, all parameters, the machine, etc. may only be changed with explicit permission. In the case of major or long-term changes, the testing and the proof that no quality loss occurs must be repeated. This is done through an extensive and expensive LCF (Low-CycleFatigue) sample program, which proves the necessary fatigue strength of the components.

After considering these requirements, there is only one alternative to capturing and processing data: Edge Computing. Only in this way it is ensured that even higher data rates and quantities have no influence on the performance of the machine and thus on the quality of the finished component. Because many edge solutions are managed centrally via a cloud, this poses another challenge for IT security. Since military engine components are manufactured at MTU, all data is subject to stringent safety requirements. It is therefore not possible to exchange data with an external cloud or to maintain or operate components of a standard machine from an external point of view. All that remains is therefore the possibility of an internal cloud which is not communicated to an external source at all or only under strict supervision. The requirements for a complex process data management demand, in contrast to the current state of the art:

\section{- Increased accuracy and data rate}

- $\quad$ No impact on the NCU

- Time-synchronized integration of external sensors and machine data

The last point has to be clarified for each process step in relation of which combination and which method of data processing generates the best results for the data monitoring.

In order to reproduce the process in its entirety, it is currently being attempted to equip new machines with external sensors and to combine these with the machineinternal data. For series operation, it would be desirable to be able to map as much as possible of the process via the machine-internal data, since these are easier to acquire and evaluate. In addition, the integration effort compared to external sensors is also modest. Here, however, often arise difficulties with the machine or control manufacturers. There are currently few mature concepts for the mass production to describe complex machining processes entirely from machine-internal data. With increased demands on an inmachine process data management, most manufacturers also face great development effort and require experience from the field. The positioning of external sensors by the manufacturer is also new territory when it comes to more complex monitoring of the process. In this case it is important to collect practical experience and to develop suitable concepts together.

Subsequently, in serialization, such concepts should be able to be integrated in the machine order, and the software maintenance should additionally be carried out by the manufacturer. This integration is made considerably more difficult by a diversified machine park, by the different manufacturers and their different approaches in the handling of these tasks. Different machines often come from different generations and thus often contain different generations of machine control, for example the Siemens Powerline or Solutionline, which provides different conditions for the connection of external hardware and software. However, the operability of the process data management solution should be easy for the user, regardless of the generation of the controller or machine, and imply as little effort as possible for the operator, so the operation of the process is not disturbed and the productivity does not suffer. The evaluations should be either adjusted or done directly in the background on the machine.

In addition to recording and processing, the evaluation in the series environment should also be automated in order to relieve the operator and provide him with only the quality data relevant to him. This allows the operator to analyze the data close to the machine to quickly make a decision on the quality of the component. If the quality data is transferred from the machine to a QA (Quality Assurance) system, a broader database can be built up over a longer period of time, allowing analysis across multiple components to ensure consistent component quality and monitor the condition of the machine.

In order to be able to combine the data into a database, it is necessary that different manufacturers use uniform open 
interfaces in order to be able to act as a complete system within a company. OPC UA, for example, could be such an interface, but higher-frequency data can be a problem in this case. Thus it must be decided for the particular application which interface is suitable. After successful data acquisition, the creation of a uniform data structure and storage takes place. The differences described above and the different sample rates for different internal and external sensors result in extremely different file sizes and formats. Nevertheless, these data have to be made stable for a valid analysis and made available in a uniform format.

MTU is currently working on two approaches to process data management. On the one hand, in collaboration with a control manufacturer, the configuration of an IPC (Industrial-PC) with non-reactive data acquisition and synchronization with analogue data sources should be worked out, which will be implemented with advanced algorithms for process quality assessment of components. On the other hand, external data sources are investigated, which should be time-synchronized with the internal data. For this purpose, different sensor types and their positioning in facilities of machining production are tested. The raw signals of the sensors are considered, analyzed and evaluated with statistical methods as well as the necessary process knowledge. Here, characteristic values are to be generated which facilitate the future evaluation and save resources, since only these values are stored as quality characteristic value in the long term. The individual processing steps during the production of a component are to be clustered in order to make the data comparable and better analyzable. This makes it easier to identify trends and anomalies for faster and more efficient processing and removal.

\section{CONCLUSION}

Due to the special, difficult-to-machine materials, the highpriced and dynamically highly loaded components, the strict regulations for component approvals as well as the high quality assurance costs, which can account for up to $30 \%$ of the selling price of rotating components of the aircraft gas turbine, the acquisition and analysis of process data becomes necessary high demands. The data must be collected during the process, in some cases in real time. Although the focus is on machine-internal data, external sensors with a uniform time stamp are also considered. An overview of a possible solution structure for Edge Computing for a manufacturing process is shown in Figure 4.

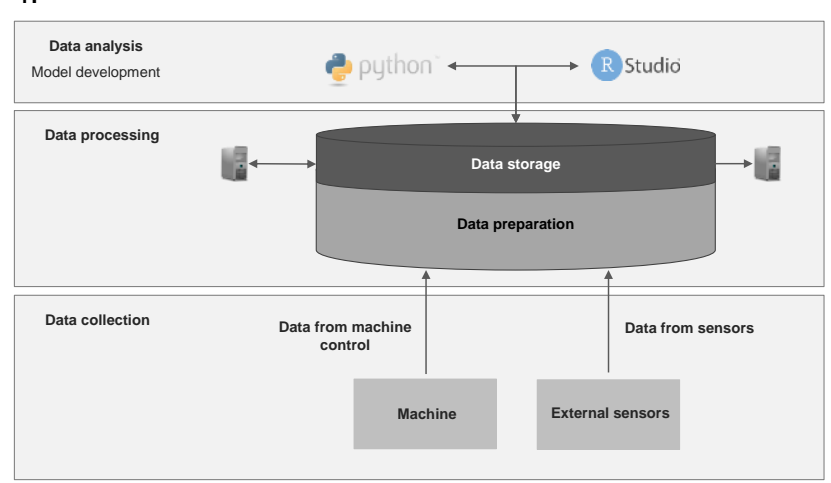

Fig. 4: Overview of a potential Edge Computing solution for a manufacturing process.

On the basis of the presented production example, milling on a thin-walled compressor blisk, in which the determined process data must also be assessed continuously from the point of view of the changing modal situation, it becomes clear that a very high amount of data has to be evaluated parallel to the process. With the bandwidth available in a networked factory today, processing in the form of Edge Computing is required. The success factors mentioned in this work are essential for the development of a functioning process data management in aviation. The goal is an early process statement if the quality of the workpiece is in order or not. In the future, a statement about the machine state during processing and in the distant future the preventive process control shall be made.

In the context of the EU project Accent it was proved that in principle a preventive process control is possible during turning and drilling. However, this project also showed that the potential for controlled manufacturing in an industry such as the aerospace industry, with a globally networked supplier system, can only be optimally utilized if the results lead to a global standard.

\section{REFERENCES}

[Bräunling 2009] Bräunling, W., J. G.: Flugzeugtriebwerke, Springer Verlag, Berlin.

[Eckstein 2013] Eckstein, M.: Monitoring microstructure and residual stresss in hole-making of Inconel 718, Dissertation TU Kosice

[Gunston 1996] Gunston, B.: Jane's Aero Engines, Jane's Information Group, ISBN0710614055, , ISBN0710614056

[Hofmann 2017] Hofmann, E.; Rüsch, M.: Industry 4.0 and the current status as well as future prospects on logistics. In: Computers in Industry, 2017, Volume 89. Page 23-24.

[NTS 1996] N.N.: aircraft Accident Report - Uncontained Engine Failure Delta Airlines Flight 1288, National Transportation Safety Board, Washington D.C.

[Reinhardt et al. 2012] Reinhardt, S.; Fischl, M.; Reinhart, G.: Characterization and Weighting Scheme to Assess the Resource Efficiency of Manufacturing Process Chains. In: Proceedings of the 19th CIRP Conference on Life Cycle Engineering, 2012. Page 509-514

[Shi et al. 2016] Reinhardt, S.; Fischl, M.; Reinhart, G.: Edge computing: Vision and challenges. In: IEEE Internet of things journal, Vol. 3, No. 5, 2016. Page 637-646.

[Tönshoff 2002] Tönshoff, H.K.; Friemuth, T.; Becker, J.C.: Process Monitoring in Grinding. In CIRP Annals, Volume 51, Issue 2, 2002. Page 551-571.

[MTU 2019] MTU Mediapool 2019 